\title{
Climate connections
}

\author{
The climate crisis highlights just how connected the world is. But understanding the changes cascading throughout \\ the natural world calls for even greater connectivity: between countries, scientists and scientific disciplines.
}

T he field of ecology has long involved studies of human impacts on the natural world, but the ongoing climate crisis brings a new sense of urgency into play. The nature of the crisis - which persists globally and impacts organisms ranging from microbes to megafauna highlights the need to take a multi-scale, multi-disciplinary and multinational approach in order to understand and support the changing world.

Although ecology has historically been dominated by studies focused on changes at the population and local community levels, there is a growing and welcome trend to interrogate climate changes across all biological scales: from the subcellular to the global. At the molecular scale, climate impacts at the level of genes, proteins and metabolites, while well studied in model organisms and certain crop species, remain underrepresented. Nonetheless, it is increasingly clear that inter- and intraspecific genetic variation are key components in defining the susceptibility of populations, species or entire ecosystems to global change. Furthermore, there is growing evidence that natural (for example, Heider et al. ${ }^{1}$ ) or induced (for example, Buerger et al. ${ }^{2}$ ) genetic variation may be exploited as a pathway to climate change resilience. Such studies demonstrate how genetic variation can have an impact beyond the level of the individual, and underscore the value of increased incorporation of molecular biology approaches into the climate change ecology field.

At the other end of the ecological scale, global- or broad-scale studies have rapidly increased, aided by remote sensing technology advances. Some limitations remain; global data should ideally be supported by in situ studies, yet these continue to be geographically biased, with a dearth of research in the very regions that face the highest climate risk ${ }^{3}$.

This geographic bias seems to be reflected in researcher connections: infometric analysis of highly cited environmental science papers shows

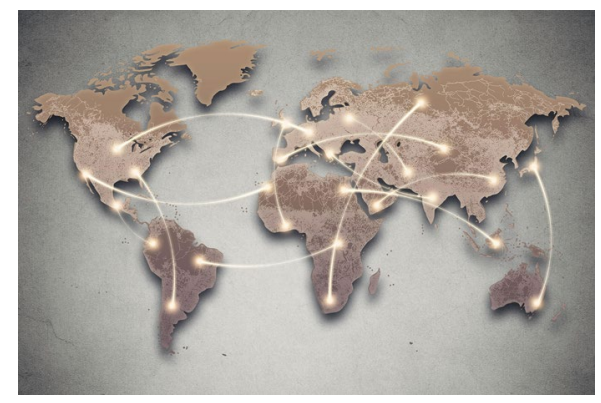

Credit: Ivan Chiosea / Alamy Stock Photo

that strong collaborative networks between the USA and various European countries still dominate ${ }^{4}$. Recent travel restrictions have revealed the potential for virtual connections, which can aid the democratization of science, and, with a concerted effort, facilitate divergence from these historical networks. Optimistically, this 'virtual shift' can be further exploited to unite disciplines by facilitating access to meetings and seminars that might otherwise be seen as too specialized to justify the expenditure of time, energy and money.

As such, it seems increasingly possible to envisage climate change research that spans disciplines, countries and biological scales. However, connecting different datasets into complex models that represent and ultimately predict the changing world remains a further challenge.

In recent years, there have been calls to increase the inclusion of various biotic mechanisms and interactions into models predicting organism responses to change ${ }^{5}$. One example of such a study, which explicitly incorporates the multi-level reciprocal interactions between species and abiotic factors within a given ecosystem, is in this issue. Greenspan and colleagues use miniature ecosystems - those existing within the liquid-filled tanks of bromeliad plants - to investigate the ecological consequences of warming at multiple biological scales (macro- and microorganisms), and reveal unexpected direct and indirect links between biotic and abiotic changes.
Still, while disentangling climate connections may be possible for simplified ecosystems such as the bromeliad tanks, it remains largely inaccessible in more complex contexts for all but the most well-studied species. Furthermore, detailed mechanistic models rely heavily on the incorporation of species- or community-specific information, which in turn can limit the applicability of such models beyond the organisms in question.

These limitations have resulted in the development of unique approaches. One recent method, termed 'rescaling ${ }^{6}$, involves collapsing multiple climate variables into a single biological factor, which is then used as a climate proxy for predicting broader community changes. A second simplifying approach involves moving the focus away from species and instead investigating functional traits. For example, Yitao Wang and colleagues ${ }^{7}$ showed the unifying impact of acidification on motility across diverse flagellated algae species, with potential implications for a range of flagellated cell types found in different kingdoms of life.

Although the value of these approaches has yet to be tested broadly within the field, an intriguing feature is that the key to the success of both methods involves the willingness to change perspective. This, in turn, may be part of the broader message for climate science connectivity - that we are reaching a stage where we are as much limited by expectations as by tools and technology.

Published online: 27 October 2020 https://doi.org/10.1038/s41558-020-00947-x

References

1. Heider, B. et al. Nat. Clim. Change https://doi.org/fc7p (2020).

2. Buerger, P. et al. Sci. Adv. 6, eaba2498 (2020).

3. Feeley, K. J., Stroud, J. T. \& Perez, T. M. Biodivers. Lett. 23, 231-234 (2017).

4. Ma, Q. et al. Int. J. Environ. Res. Public Health 17, 3781 (2020).

5. Urban, M. C. et al. Science 353, aad8466 (2016).

6. Vandvik, V. et al. Proc. Natl Acad. Sci. USA 117, 22858-22865 (2020)

7. Wang, Y. et al. Nat. Clim. Change 10, 561-567 (2020). 